\title{
Le genre discursif comme objet d'enseignement en didactique du français
}

\author{
Claudel, Chantal \& Laurens, Véronique \\ Université Paris 8 - EA 7345 - CLESTHIA (Université Paris 3) \\ Université Sorbonne Nouvelle Paris 3 - EA 2288 - DILTEC \\ chantal.claudel@univ-paris8.fr \\ veronique.laurens@univ-paris3.fr
}

Résumé. Le tournant communicatif des années 1970 a marqué l'évolution des travaux en didactique du français en orientant l'enseignement de la langue à partir de l'étude de documents et de contextes de communication variés. Dans cette perspective, les objets langagiers privilégiés sont le genre discursif et l'acte de parole. Si en didactique du français langue première (FL1), l'accès à la langue semble s'effectuer principalement par l'entremise des genres, en didactique du français langue étrangère (FLE), l'entrée privilégiée passe essentiellement par le recours aux actes de parole, sans pour autant que la notion de genre ne soit exclue des préoccupations du champ. Cet arrière-plan épistémologique laisse entrevoir l'existence de points de divergence, mais également de lieux de rencontre entre les deux contextes. Ceux-ci ont en effet en partage un même objet, le français et une même notion, le genre. Dans les deux cas, le genre est appréhendé comme objet d'enseignement-apprentissage, bien que l'importance de cette entrée demeure encore assez secondaire en didactique du FLE au regard de la place qui lui est accordée en FL1. Pour autant, où se situe la ligne de démarcation entre le genre en FL1 et le genre en FLE ? En outre, lorsqu'on opère un resserrement sur les pratiques en FLE, comment cette entrée est-elle appréhendée ? Ce sont ces deux questions qui sont abordées dans cet article à travers un état des lieux des positionnements privilégiés en FL1 et en FLE et la présentation de pistes d'exploitation des genres en FLE.

\begin{abstract}
Since the communicative orientation of the 1970s in French language teaching, texts, documents and situations have been used to contextualize language learning. In this perspective, discursive genres and language functions have been established as the main language objects to be taught. In French as a first language (FFL), French is mainly studied through discursive genres, whereas in French as a (foreign or) second language (FSL), language functions are primarily used. This epistemological background shows similarities and differences between the two domains dedicated to the teaching of French. They share the same object, French, and they have a notion in common, the discursive genre. In both fields, the discursive genre is approached as a teaching/learning object, although it comes second in FSL compared to its importance in FFL. What distinguishes the approach of genre in FFL and FSL? How is genre used in FSL teaching? These are the two questions that will be discussed in this paper, based on a review of positions in FFL and FSL, and the presentation of various pedagogical uses of discursive genres in FSL.
\end{abstract}


Le tournant communicatif des années 1970 a profondément marqué les évolutions de l'ensemble des travaux en didactique du français en mettant l'accent sur la nécessité d'aborder l'enseignement de la langue de manière intégrée. Cette orientation s'est développée notamment en prônant l'articulation du travail sur la langue et l'étude de documents et de contextes de communication variés afin de faire prendre conscience des formes langagières à utiliser dans diverses situations. Les objets langagiers privilégiés depuis lors pour aborder la langue sont le genre discursif et l'acte de parole. Si en didactique du français langue première (FL1), l'accès à la langue semble s'effectuer principalement par l'entremise des genres, en didactique du français langue étrangère (FLE), l'entrée privilégiée passe essentiellement par le recours aux actes de parole (cf. Dolz et al. 2015), sans pour autant que la notion de genre ne soit exclue des préoccupations du champ (cf. notamment Beacco et Darot 1984, Beacco 2004, Moirand 1990, Peytard et Moirand 1992, Claudel 2009, Truong 2010). Néanmoins, dans le domaine du FL1, les genres ont été abordés dans le cadre des recherches sur l'interactionnisme socio-discursif (Bronckart et al. 1985, Bronckart 1996) et articulent la problématique sur leur enseignabilité dans le contexte scolaire, tandis qu'en didactique du FLE, cette entrée s'est opérée à la faveur du concept de compétence de communication élaboré par D.-H. Hymes (1972), lequel est à l'origine de la mise en cause de la seule dimension linguistique dans les situations d'échanges (cf. modèle SPEAKING, Beacco 2013 : 189). Cet arrière-plan épistémologique laisse entrevoir l'existence de points de divergence, mais également de lieux de rencontre entre les deux contextes. Ceux-ci ont en effet en partage un même objet, le français et une même notion, le genre. Dans les deux cas, le genre est appréhendé comme objet d'enseignementapprentissage, bien que l'importance de cette entrée soit moindre en didactique du FLE comparativement à la place qui lui est accordée en FL1. On peut donc se demander où se situe la ligne de démarcation entre le genre en FL1 et le genre en FLE. En outre, lorsqu'on opère un resserrement sur les pratiques en FLE, comment cette entrée peut-elle être appréhendée ?

C'est pour répondre à ces questions que l'on se propose d'abord de mettre en perspective l'entrée dans la langue par les genres en didactique du FL1 et du FLE et que l'on introduit, dans un second temps, des pistes envisageant un traitement détaillé de l'abord des genres en FLE.

\section{Mise en perspective de l'entrée genre en didactique du FL1 et du FLE}

L'hypothèse bakhtinienne de l'expérience immédiate du langage faite à travers les genres discursifs est sous-jacente à la fois aux didactiques du FL1 et du FLE et explique le choix fait, dans ces deux champs du français, d'aborder le travail sur la langue à partir de cette entrée générique :

\begin{abstract}
[...] les formes de la langue et les formes des types d'énoncés, c'est-à-dire les genres $\mathrm{du}$ discours, s'introduisent dans notre expérience et dans notre conscience conjointement et sans que leur corrélation étroite soit rompue [...]. Les genres du discours organisent notre parole de la même façon que l'organisent les formes grammaticales (syntaxiques). (Bakhtine, 1984 : 285)
\end{abstract}

Néanmoins, les ancrages épistémologiques de l'entrée par les genres dans ces didactiques sont distincts. Nous développons ci-après l'ancrage dans l'interactionnisme socio-discursif pour l'entrée par les genres en FL1 et l'ancrage dans la notion de compétence de communication pour l'entrée par les genres en FLE. 
Cette différenciation permettra ensuite d'éclairer comment chaque tradition envisage la place de l'individu et du collectif dans le discours et débouchera sur une mise en regard des modélisations didactiques mises au point pour l'enseignement de la langue en FL1 et en FLE.

\subsection{Le genre en didactique du FL1 : ancrage dans l'interactionnisme socio- discursif}

Dans son ouvrage phare de 1996, Bronckart pose l'interactionnisme socio-discursif en ces termes : dans le sillage de la psychologie vygotskienne, ce courant psycholinguistique a pour objet de « saisir les actions humaines dans leurs dimensions sociales et discursives constitutives » (ibid. : 30). Ce courant s'appuie sur les travaux de Bakhtine qui considère l'interaction verbale comme « la véritable substance de la langue » (1977: 135), où l'énonciation ne peut être vue comme isolée mais comme constitutive de l'interaction, et où des " types relativement stables d'énoncés » sont appelés genres de discours (1984: 265). L'interactionnisme socio-discursif spécifie la notion de « genre » par rapport à celle de « texte »: les textes sont « des produits de l'activité langagière en permanence à l'œuvre dans les formations sociales » (1996: 137), les « caractéristiques relativement stables » des textes font qu'on peut les qualifier de genres de texte (ibid.). En ce sens, tout texte produit relève d'un genre. Les régularités linguistiques attestées dans les genres de textes sont considérées comme « des types linguistiques, c'est-à-dire comme des formes spécifiques de sémiotisation ou de mise en discours », que Bronckart qualifie de types de discours (ibid. 139).

La distinction entre genre de texte ou de discours et type de texte a généré un débat théorique important, notamment entre Adam et Bronckart. Les travaux d'Adam en linguistique textuelle l'ont amené à poser le texte comme objet d'étude proprement linguistique, à la différence des genres de discours, présentant des variations liées à leurs conditions socio-historiques de production (1990 : 20). Adam a ainsi tenté d'établir des typologies de textes (1992a). A contrario, Bronckart envisage les textes comme des « formes communicatives globales et finies [...] qui se distribuent en genres » (1996:150); ces produits concrets ne peuvent être considérés en dehors des genres auxquels ils se rapportent. Adam a finalement avancé que le texte était une « unité trop complexe pour être enfermé dans des typologies » (2002:571) en rejoignant l'hypothèse que les régularités formatives des textes sont liées aux genres de discours (ibid.).

L'hypothèse bakhtinienne d'appréhension de la langue par les genres discursifs est opérante et justifie l'entrée unique par ce biais en didactique du FL1, car, dans une culture donnée, c'est par les genres qu'il y a communication et action (langagière) ; l'apprentissage de la langue passe alors par l'appropriation des outils qui permettent d'agir langagièrement, c'est-à-dire les genres (Dolz \& Schneuwly, 1998 : 64-65). Le genre, défini par des contenus, des structures communicatives et des traits linguistiques, a aussi pour avantage de représenter des formes langagières identifiables et reconnues dans l'infini des réalisations possibles de la langue, ce qui donne un cadre d'apprentissage aux apprenants.

Par rapport à l'extrême variabilité des pratiques langagières, les genres peuvent être considérés comme des entités intermédiaires permettant de stabiliser les éléments formels et rituels des pratiques. Ainsi le travail sur les genres dote les élèves de moyens d'analyse des conditions sociales effectives de production et de réception des textes. Il fournit un cadre d'analyse des contenus, de l'organisation de l'ensemble du texte et des séquences qui le composent, ainsi que des unités linguistiques et des caractéristiques spécifiques à la textualité orale. (ibid. : 65)

\subsection{Le genre en didactique du FLE : ancrage dans la notion de compétences de communication}

En didactique du FLE, les années 1970 marquent l'avènement de l'approche communicative. Centrée sur l'apprenant, soucieuse de la prise en compte de ses besoins, ou encore préoccupée par les paramètres de la situation de communication (Hymes 1972), cette approche va largement se nourrir des avancées de la pragmatique et en particulier, de la notion d'acte de langage que la didactique du FLE va transformer " en unité d'enseignement»(Beacco 1980:37 ; Peytard et Moirand 1992 : 48). Cette conception de la 
communication conduit à prendre en compte non plus uniquement un savoir linguistique, mais également «[...] un savoir sociolinguistique ou, en d'autres termes, une connaissance conjuguée de normes de grammaire et de normes d'emploi. » (Hymes 1984:47) De cette évolution vont naître de nouvelles démarches méthodologiques. L'émergence de l'importance accordée aux documents authentiques, plus à même de répondre aux attentes des différents publics, est à situer à cette période. Leur sélection et leur pédagogisation vont notamment impliquer la prise en compte de catégories issues de la pragmatique. L'intérêt manifesté pour ce champ de recherche et plus spécifiquement, pour les actes de langage en didactique du FLE est aussi à rapporter aux pratiques pédagogiques antérieures à l'approche communicative. Dans la lignée des propos de Besse (1980a : 46), Richterich note que l'une des critiques faites à des méthodes comme les méthodes structurales-behavoristes audio-visuelles «était qu'elles imposaient à chacun les mêmes façons d'apprendre un modèle de langue identique présenté dans un nombre restreint de situations stéréotypées. » (1985:25) L'introduction de la notion de besoin langagier au début des années 1970 va engendrer un recentrement sur la personne de l'apprenant pour en faire un individu « $[\ldots]$ en grande partie responsable de son propre apprentissage, dans la mesure où le rôle de l'enseignant est moins directif ou autoritaire que dans les méthodes ou approches traditionnelles. » (Germain 1993 : 206) Parallèlement, l'on voit comment «sous la pression des besoins (économiques, politiques, touristiques, etc.) de la société contemporaine», on établit «la nécessité d'enseigner les langues secondes comme instruments de communication. » (Roulet $1980: 81$ )

Plusieurs courants de recherche ont favorisé l'émergence de cette conception de l'enseignement. Les travaux menés en sociologie - à qui l'on doit les notions de statut et de rôle (cf. Coste 1980a : 26) - et en sociolinguistique (cf. Goffman 1974, Hymes 1972, Labov 1966) notamment ont contribué à éveiller l'attention sur l'existence de groupes sociaux ou encore, de contextes socio-relationnels, professionnels, culturels, etc. variés. Dans ce cadre, l'acquisition d'une compétence de communication renvoie à la capacité d'adapter ses dires, ses manières d'être et/ou de faire à l'environnement concerné. A contrario, il revient au pédagogue de mettre en œuvre des approches - en termes de progression, de démarche, de supports, etc. -, adaptées à la diversité et aux besoins des publics auxquels est destinée la formation en langue.

Le public en FLE n'est pas, comme en milieu scolaire, un public défini à l'avance, ni même un public captif (cf. Courtillon 2003 : 19-20) tenu de suivre un enseignement où les besoins et les objectifs sont préalablement déterminés par des circulaires ministérielles. Les contenus qui lui sont destinés sont le fruit d'une réflexion menée à l'issue de l'analyse des besoins langagiers ${ }^{1}$. Ils s'articulent autour d'actes de communication $^{2}$, lesquels impliquent la prise en compte de « la diversité et de la complexité des utilisations langagières dans leurs contextes réels, de façon non pas universelle mais par rapport, d'une part, à leurs caractéristiques, d'autre part, à celles des utilisateurs et des apprenants. » (Richterich op. cit.) Ces orientations marquent le passage d'un enseignement-apprentissage s'appuyant sur des techniques uniformisées, tourné vers un public indéterminé à des actions ciblées, nécessitant du sur mesure. Il s'en suit une démarche attachée à «différencier les enseignements par rapport à des individus ou groupes d'individus déterminés afin qu'ils apprennent une langue étrangère dans des conditions qui leur soient propres. » (ibid. 26)

Ce regard porté d'une part, sur le profil du public (centration sur l'apprenant) et sur la nécessité de lui faire acquérir une compétence de communication en adéquation avec ses besoins langagiers, et d'autre part, sur l'importance, pour l'enseignant, de définir des objectifs d'apprentissage liés à ces variables, donne toute sa cohérence à la prise en considération d'une catégorie descriptive comme celle d'acte de langage. Cette entrée s'inscrit dans le prolongement des approches énonciatives elles-mêmes tournées vers l'examen

non seulement [des] structures phonologiques, grammaticales ou lexicales de l'énoncé, mais également des conditions de production, à savoir le statut relatif des interlocuteurs, l'acte de communication visé, les intentions sous-jacentes. (Verdelhan $1982: 108)$

Ainsi, la conception selon laquelle tout énoncé est à saisir dans sa relation avec les conditions de production desquelles il émane est déjà en place. Se situant à un degré de conceptualisation plus élevé que 
les marqueurs linguistiques, l'acte de langage va occuper une place de choix en didactique du FLE (cf. Un Niveau Seuil 1976). Le découpage que cette catégorie pragmatique permet d'opérer en termes d'objectif ou encore de progression, en fera une pièce maîtresse du champ. Parallèlement, la nécessité de didactiser des documents en adéquation avec des profils d'apprenants variés engendre la description de données langagières de dimension supérieure à la phrase (énoncés, textes, interaction). C'est dans ce contexte que s'inscrit le recours à l'énonciation indicielle et ce faisant, à l'analyse de discours (désormais AD) (cf. Moirand 1979 : 13-16 et 1990a).

Le choix de l'une ou de l'autre de ces orientations disciplinaires est fonction de la nature des textes supports et définit des approches différentes. Alors que l'entrée pragmatique privilégie des corpus oraux se référant à une langue usuelle traitée dans une perspective onomasiologique, l'entrée par le biais de l'AD est davantage tournée vers l'approche de textes écrits, recourant à une langue de spécialité abordée dans une perspective sémasiologique (Coste 1980b : 244). Mais les rapports de l'AD avec les données authentiques ont évolué. Depuis les années 1995-2000 en effet, la discipline est tout aussi attentive aux échanges oraux qu'aux écrits produits notamment en situation professionnelle (Mourlhon-Dallies 2008 : 114).

\subsection{Perspectives croisées entre FL1 et FLE}

Au terme de cette comparaison de la détermination du genre comme objet d'enseignement en FL1 et en FLE, il ressort que les deux approches ont en commun de faire aborder les savoirs et les savoir faire langagiers à partir du traitement de documents afin de faire produire des genres scolaires/sociaux en FL1 ou afin d'amener les apprenants à (inter)agir en situations de communication en FLE. Ce qui les différencie se situe au niveau de l'ancrage théorique dans la théorie des genres. En FL1, l'ensemble de la démarche est conçu comme une émanation didactique et pédagogique de l'interactionnisme sociodiscursif. En FLE, la théorie des genres est l'une des sources à la base de la détermination des savoirs et des savoir faire langagiers ciblés dans le cadre d'une approche communicative/actionnelle. Le croisement avec d'autres théories linguistiques (pragmatique, énonciation, sémantique) nourrit les manières d'aborder deux types d'objets d'enseignement, le genre discursif et l'acte de parole. Le travail sur ces deux objets permet de développer la compétence à communiquer langagièrement des apprenants.

Par ailleurs, il se dégage des positionnements adoptés en FL1 et en FLE, deux visions distinctes de l'apprenant: l'une l'appréhende comme une entité prédéterminée, assujettie à la collectivité, l'autre comme un sujet individuel à identifier précisément à chaque nouvelle formation (cf. supra 1.2.). Un tel cadrage induit une différence de conception dans la façon même de se saisir des objets à introduire en classe. Dans le cadre de l'enseignement/apprentissage scolaire des disciplines, les objets d'enseignement sélectionnés sont organisés en programmes décrits dans les instructions officielles. L'approche implique des pratiques harmonisées dans le mode de saisie des savoirs et des savoir-faire sur la base de principes organisateurs destinés au plus grand nombre. À ce niveau, l'élève est cerné du point de vue du collectif qu'il représente. Il en va différemment dans le domaine du FLE hors contexte scolaire en France, puisque l'une des spécificités consiste en l'absence de programmes de formation prédéfinis. Cette absence de programmes est la garantie d'une souplesse d'adaptation aux besoins de l'apprenant et ce faisant, à ceux d'un individu bien circonscrit.

Cette brève synthèse fait ressortir une conception partagée, en FL1 et en FLE, dans le traitement de documents pour la classe, lesquels sont appréhendés comme un moyen de mettre en œuvre des pratiques situées. Elle souligne en outre des lieux de divergences dans la manière dont les champs du FL1 et du FLE s'ancrent épistémologiquement et dans la perception qu'ils ont de l'apprenant. La différence d'ancrage épistémologique entre didactique du FLE et didactique du FL1 trouve son prolongement dans les modélisations didactiques construites pour l'enseignement de ces objets (Laurens 2012).

En langue étrangère, l'unité didactique est conçue pour réaliser une tâche ou communiquer en situation à partir de l'appropriation d'actes de langage ou la compréhension/production d'un genre par le traitement d'un (ou de) texte(s) : il y a pluralité des sources d'unités didactiques, la diversité des tâches, des situations et des genres étant liée à la grande variabilité des pratiques langagières (informelles et 
formelles) à faire travailler aux apprenants, en fonction des besoins et des contextes d'apprentissage dans lesquels ils se trouvent. Les caractéristiques pragmatiques, socio-linguistiques/culturelles et linguisticodiscursives des textes sont recensées à l'aide de la grille d'analyse pré-pédagogique (Moirand 1979 ; Lebre-Peytard 1990).

En langue première, la séquence didactique est construite pour s'approprier un genre oral ou écrit formel enseignable dans le cadre scolaire et garant en lui-même de l'unité de la séquence didactique (cf. De Pietro et Dolz 1997, De Pietro, Erard et Kaneman-Pougatch 1996/97, Schneuwly et Dolz 1997). Les textes sélectionnés comme supports sont tous des exemples d'actualisation du genre ciblé qui agissent comme des documents de référence ou des exemples de réalisation. A partir de modèles didactiques des genres discursifs sélectionnés pour l'enseignement/apprentissage scolaire (Dolz et Schneuwly 1998, De Pietro et Schneuwly 2003), les apprenants étudient les textes appartenant au genre étudié et s'approprient les activités langagières qui lui sont spécifiques. Des séquences didactiques ont été conçues pour l'enseignement de genres scolaires oraux (Dolz et Schneuwly 1998). Puis, la démarche a permis de développer des moyens d'enseignement équivalents pour l'écrit (Dolz, Noverraz et Schneuwly 2001)3.

\section{Modes de traitement du genre en FLE}

Comme on l'a introduit supra, en FLE, les savoirs et les savoir faire langagiers ciblés à partir des textes sources relèvent pour l'essentiel de deux notions: l'acte de parole et le genre discursif. Cette démultiplication des points d'entrées dans les documents est liée à la complexité du concept de compétence de communication, d'une part, et aux différentes théories linguistiques dont les didacticiens se sont inspirés pour élaborer une approche communicative de l'enseignement des langues, d'autre part. Malgré les travaux importants développés pour la prise en compte de la dimension discursive et textuelle des documents supports, notamment en vue de l'élaboration de programmes de formation (Peytard et Moirand, 1992: 86), cette orientation est longtemps restée minoritaire dans les démarches d'enseignement/apprentissage, la focalisation sur les actes de parole obstruant la dimension discursive (Beacco, 1989 : 145). Et si elle demeure encore peu présente dans les manuels, elle est au centre de certaines préoccupations de recherche comme on va l'observer.

\subsection{Du document authentique au genre}

En didactique du FLE, l'importance du document authentique est avérée quelle que soit l'approche privilégiée : approche communicative ou perspective actionnelle. Ce type de support occupe une place centrale dans le dispositif de formation en langue. Tout en permettant à l'apprenant de mieux comprendre les contextes d'utilisation de l'idiome concerné, il l'expose à une langue située et à des traits culturels proches de ceux qu'il est susceptible de rencontrer en situations quotidiennes, les contenus véhiculés dans les documents retenus pour la classe ne subissant aucune modification ${ }^{4}$. Leur sélection par les enseignants peut partir de phénomènes linguistiques, discursifs ou pragmatiques récurrents. Néanmoins, penser la démarche de sélection en se fondant sur le genre discursif ouvre des perspectives plus larges et plus "guidantes" que ne le permet une approche par des phénomènes spécifiques. Tout genre véhicule un ensemble de caractéristiques ancrées dans la réalité institutionnelle, académique, professionnelle, etc. des communautés qui en font usage. Et pour cause, à « chaque type de pratique sociale correspond un discours qui se divise en genres textuels oraux ou écrits. » (Rastier 2001 : § 26). Toute production (écrite ou orale) est donc une performance linguistique (ibid. § 19) en prise avec des types d'actions situées. À ce propos, le rapprochement opéré par ailleurs entre le genre interview de presse en français et en japonais et les pratiques journalistiques à l'œuvre dans les deux communautés a permis d'illustrer cette influence des pratiques sociales, professionnelles, institutionnelles, etc. sur la construction des discours (Claudel 2004 : $28-33$; 2010a).

La connaissance de ce marquage social peut s'avérer essentielle pour la compréhension et la production de discours en langue étrangère. La démarche permet de rapporter le contenu des supports d'apprentissage relevant d'un genre à des lieux et à des conditions d'emploi relativement prévisibles. 
Cette contextualisation peut constituer une aide précieuse pour décoder le sens d'une unité linguistique, pour déduire la visée illocutoire d'un énoncé, ou encore pour accéder à des facteurs socio-relationnels ou culturels à l'origine de l'utilisation de telle ou telle configuration. Elle l'est aussi pour la construction de textes écrits ou d'énoncés oraux, les matrices du genre de référence proposant un modèle auquel il est possible de se rapporter.

Dans le cadre de formations à la didactique des langues, le recours à la notion de genre discursif permet un travail de réflexion sur les rapprochements envisageables entre documents quelque peu similaires en raison des configurations discursives et des phénomènes extra-linguistiques (environnement, situation de communication, jingle, décors, etc.) - ou para linguistiques (prosodie, kinésie, gestuelle, typographie, etc.) propres au genre qui les caractérise. Ces traits génériques permettent de mieux poser la distinction entre par exemple une fiche conseil et un mode d'emploi.

\subsection{Une entrée économique sur le plan cognitif}

Le genre permet aussi une «économie cognitive» (Maingueneau 2002: 49) non négligeable. La connaissance préalable des mécanismes qui régissent le fonctionnement des documents qui en relèvent, permet aux enseignants de faire anticiper certaines de leurs caractéristiques. Cette connaissance fine des configurations propres à un genre aide à la sélection rapide de supports appropriés et à leur renouvellement fréquent.

La proximité entre supports peut en outre être dégagée de phénomènes extra ou para linguistiques de type iconique (illustrations, schémas, croquis, encadrés, etc.), prosodique, kinésique, gestuel, etc. Ainsi, l'identification du genre "interview de presse" repose sur la reconnaissance de certains aspects de cet article médiatique construit autour de la mise en scène d'un interviewé et d'un intervieweur. Cette configuration suppose l'introduction de citations et/ou de structures de dialogues mises en relief par des procédés typographiques spécifiques de la communication imprimée (guillemets, tirets, changement de graisse de caractères, etc.). Au niveau linguistique, la mise en scène des participants de l'interview passe par l'utilisation de marqueurs de subjectivité grâce auxquels la figure des intervenants peut être appréhendée (Claudel 2009). Cette brève description de l'interview renvoie non seulement au genre rencontré dans les médias français et francophones, mais aussi au genre en japonais (Claudel 2004).

Le recours à des genres circulant dans la langue/culture source constitue un facilitateur pour l'apprenant : le document offre un degré d'anticipation élevé sur la situation d'énonciation et la possibilité d'hypothèses prévisibles sur sa visée pragmatique; de la sorte, son appréhension s'en voit simplifiée ${ }^{5}$.

L'expérience de l'apprenant en tant que locuteur natif favorise la mise en œuvre de stratégies permettant l'accès au sens et le transfert de ces stratégies en langue étrangère. Elle réduit également l'appréhension que tout enseignement/apprentissage peut engendrer, la démarche reposant sur du familier. En outre, c'est dans un mouvement allant du connu vers l'inconnu que se développe le processus de saisie des textes (cf. Claudel, Lemeunier 2012).

\subsection{Propositions d'entrée par les genres à l'aide des outils de l'analyse du discours}

La sélection de documents à didactiser sur la base du genre obéit à un protocole facile à mettre en place dès lors qu'il s'appuie sur des régularités matricielles aisément repérables et dont l'immuabilité permet une réactualisation rapide des supports. Le recours à l'AD offre de son côté aux enseignants la possibilité d'une entrée à multiples facettes dans les documents ainsi recueillis.

\subsubsection{Une entrée à multiples facettes}

Mobiliser un genre implique de prendre appui sur les unités linguistiques (morphologique, syntaxique, sémantique, etc.) les plus fréquemment rencontrées dans les supports. À moins que cette entrée par les 
faits de langue ne soit délaissée au profit d'un accès aux documents par la visée communicative, les actes de langage, les séquences textuelles, les opérations discursives, les phénomènes culturels, les tâches. Cette perspective fait écho à celle préconisée par Moirand qui introduit différentes approches possibles des textes : l'approche situationnelle, l'approche conversationnelle, l'approche pragmatique, l'approche énonciative, ou encore l'approche textuelle (1990b : 91-109).

$\mathrm{Au}$ traitement choisi correspond un ancrage du genre dans différents cadres théoriques. S'attacher aux fonctions ou à la visée pragmatique des supports invite à se pencher sur les travaux de Searle et d'Austin, mais aussi sur ceux de Grize (1990) si l'on envisage de remonter aux schématisations contenues dans les documents, (cf. Peytard et Moirand 1992 : 163-169). Mettre l'accent sur les régularités compositionnelles d'un document par exemple suppose que l'on fasse appel aux séquences textuelles en se référant notamment à Adam (1992b, 1999). Se préoccuper des phénomènes énonciatifs conduit presque automatiquement à convoquer les conceptions introduites par Benveniste (1966, 1974). Aborder la dimension culturelle ouvre la voie à une sensibilisation aux travaux sur les interactions en situation interculturelle (cf. Béal (2010), Kerbrat-Orecchioni (1994), Kerbrat-Orecchioni et Traverso (2008), Traverso (2001)), ou encore sur les genres discursifs dans différentes communautés (cf. Claudel (2004, 2010b, 2015), Mourlhon-Dallies (1995), von Münchow (2004, 2011)).

Cependant, aujourd'hui encore, le genre est une catégorie sur laquelle les manuels de langue s'appuient peu, si ce n'est dans Café Crème (Beacco di Giura et al. 1997). L'examen d'autres méthodes de FLE a néanmoins montré qu'un genre comme la lettre était généralement très présent ${ }^{6}$. On rencontre aussi des genres comme le courriel - au cœur du manuel Connexions (Loiseau et Mérieux 2004) - et plus sporadiquement, la brochure de voyage, le fait divers ou la conversation téléphonique ${ }^{7}$. Dans Version Originale 1 (Denyer et al. 2009) et le Nouveau Rond-Point 1 (Flumian et al. 2011), la table des contenus présente une entrée intitulée : «typologie textuelle » qui regroupe quelques genres : programme télé, brochure publicitaire, liste de courses, courriel d'invitation, etc. Cependant, ce traitement est trop ponctuel pour favoriser la diffusion du genre comme objet d'enseignement auprès des enseignants de langue.

Cela étant posé, l'attention portée aux diverses entrées proposées supra souligne l'intérêt manifesté pour une approche par le genre, mais également pour un accès dans les supports à didactiser par les faits de langue, les actes de langage, etc. comme on va l'examiner.

\subsubsection{Didactiser des objets de savoir pour la classe}

Si l'on se réfère au déroulement de la formation à la méthodologie des langues introduit par Laurens (2013), et plus précisément, pour la conception d'unités ou de séquences didactiques, notre propos se situe plus particulièrement à l'étape 1 du schéma présenté ci-dessous et renvoie à la détermination d'objets d'enseignement :

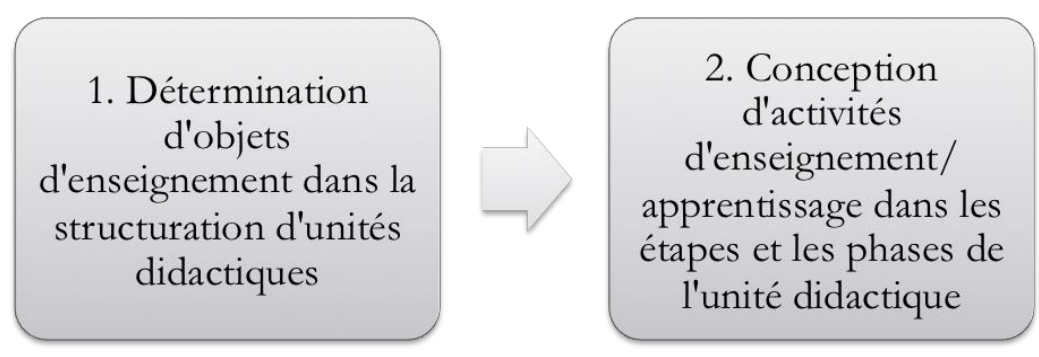

Graphique $1 \quad$ (ibid. : 218)

Lors des phases de l'analyse pré-pédagogique, il s'agit de caractériser précisément les objets à introduire en classe. Le traitement minutieux de ces objets de savoir suit la sélection de documents authentiques destinés à être didactisés. Dans ce contexte, l'entrée par les genres constitue un outil particulièrement 
productif : il permet de rassembler, dans une démarche intégrative de l'enseignement-apprentissage de la langue étrangère, des supports de natures variées.

\subsubsection{Un feuilletage d'entrées}

Les documents écrits ou oraux relevant d'un même genre discursif se caractérisent par la présence de régularités suffisamment représentatives pour permettre de les regrouper. Une fois les supports rassemblés et reconnus comme appartenant à un genre donné, l'approche consiste à identifier l'ensemble des éléments contribuant à sa définition, ce qui suppose de localiser les traits spécifiques du genre du point de vue de ses fonctions, des actes de parole qui le distinguent, des faits de langue qu'il contient, des séquences textuelles et des opérations discursives qui le constituent, de la portée culturelle qu'il est susceptible de véhiculer et de la tâche qu'il peut impliquer. Nombreux sont les travaux, menés en AD notamment, pouvant contribuer à alimenter la réflexion en vue d'établir une cartographie des genres (cf. Claudel $2014: 96)$.

À une étape ultérieure du travail, la connaissance acquise du fonctionnement d'un genre permet une entrée modulable dans les documents. Celle-ci peut se réaliser par la visée communicative, les actes de parole, les faits de langue, les configurations textuelles, les opérations discursives, les aspects culturels et/ou encore les tâches, par lesquels une remontée vers le genre peut s'opérer. Dans la logique sousjacente à l'entrée par le genre, tous ces aspects sont inter-reliés : la langue ne peut être abordée de manière désintégrée. En guise d'illustration, le tableau ci-dessous (Tableau 1) propose un premier cadrage, dégagé sur la base de l'approche exposée supra (Graphique 1), de trois genres plus ou moins bien établis : la critique d'objets culturels, le fait divers et le courrier électronique amical.

\begin{tabular}{|c|c|c|c|c|c|c|}
\hline $\begin{array}{c}\text { Visée } \\
\text { communicative }\end{array}$ & $\begin{array}{l}\text { Acte/s de } \\
\text { parole }\end{array}$ & $\begin{array}{c}\text { Séquences } \\
\text { textuelles } \\
\text { dominantes } \\
\end{array}$ & $\begin{array}{c}\text { Opérations / } \\
\text { phénomènes } \\
\text { discursifs }\end{array}$ & $\begin{array}{l}\text { Faits de } \\
\text { langue }\end{array}$ & $\begin{array}{c}\text { Dimension } \\
\text { culturelle }\end{array}$ & Tâche/s \\
\hline \multicolumn{7}{|c|}{ Critique d'objets culturels } \\
\hline $\begin{array}{l}\text { - faire lire } \\
\text { - faire voir } \\
\text { - faire acheter } \\
\text { - faire aller } \\
\text { - etc. }\end{array}$ & $\begin{array}{l}\text { - décrire } \\
\text { - donner son } \\
\text { avis } \\
\text { - informer } \\
\text { - etc. }\end{array}$ & $\begin{array}{l}\text { - descriptive } \\
\text { - argumentative }\end{array}$ & $\begin{array}{l}\text { - description } \\
\text { - comparaison } \\
\text { - critique } \\
\text { - modalité } \\
\text { appréciative } \\
\text { (évaluatifs } \\
\text { objectifs et } \\
\text { subjectifs ; } \\
\text { axiologiques...) } \\
\text { - etc. }\end{array}$ & $\begin{array}{l}\text { - adjectifs } \\
\text { - adverbes } \\
\text { - verbes } \\
\text { d'opinion } \\
\text { - comparatifs } \\
\text { - etc. }\end{array}$ & $\begin{array}{l}\text { - le cinéma en } \\
\text { France } \\
\text { - la rentrée } \\
\text { littéraire } \\
\text { - etc. }\end{array}$ & $\begin{array}{l}\text { - sélectionner } \\
\text { un DVD ou } \\
\text { un livre pour } \\
\text { l'anniversaire } \\
\text { d'un } \\
\text { camarade } \\
\text { - sélectionner } \\
\text { le film de la } \\
\text { semaine à } \\
\text { voir avec la } \\
\text { classe }\end{array}$ \\
\hline \multicolumn{7}{|c|}{ Fait divers } \\
\hline - faire savoir & $\begin{array}{l}\text { - raconter } \\
\text { un } \\
\text { événement } \\
\text { passé }\end{array}$ & $\begin{array}{l}\text { - narrative } \\
\text { - descriptive } \\
\text { - explicative }\end{array}$ & $\begin{array}{l}\text { - règles de } \\
\text { Quintilien : qui, } \\
\text { quoi, où, qd, } \\
\text { comment } \\
\\
\text { - désignation } \\
\text { - cohésion } \\
\text { (cataphore, } \\
\text { anaphore) }\end{array}$ & $\begin{array}{l}\text { - temps du } \\
\text { passé (passé } \\
\text { composé, } \\
\text { imparfait, etc.) } \\
\text { - passif } \\
\text { - discours } \\
\text { rapporté } \\
\text { - etc. }\end{array}$ & $\begin{array}{l}\text { - les médias } \\
\text { - les modalités } \\
\text { de traitement } \\
\text { d'un } \\
\text { événement } \\
\text { - les thèmes } \\
\text { qui font } \\
\text { "événement" } \\
\text { - etc. }\end{array}$ & $\begin{array}{l}\text { - préparer un } \\
\text { exposé sur le } \\
\text { traitement } \\
\text { différencié de } \\
\text { l'information } \\
\text { - organiser } \\
\text { une revue de } \\
\text { presse }\end{array}$ \\
\hline
\end{tabular}




\begin{tabular}{|c|c|c|c|c|c|c|}
\hline & & & $\begin{array}{l}\text { - modalisation } \\
\text { en discours } \\
\text { second } \\
\text { - etc. }\end{array}$ & & & \\
\hline \multicolumn{7}{|c|}{ Message électronique amical } \\
\hline $\begin{array}{l}\text { - faire savoir } \\
\text { - faire faire } \\
\text { - etc. }\end{array}$ & $\begin{array}{l}\text { - saluer } \\
\text { - s'enquérir } \\
\text { de la santé du } \\
\text { destinataire } \\
\text { - expliquer } \\
\text { l'objet du } \\
\text { message } \\
\text { - remercier } \\
\text { - s'excuser } \\
\text { - faire un } \\
\text { projet } \\
\text { - prendre } \\
\text { congé } \\
\text { - etc. }\end{array}$ & $\begin{array}{l}\text { - dialogale } \\
\text { - explicative } \\
\text { - argumentative }\end{array}$ & $\begin{array}{l}\text { - formulations } \\
\text { directes } v s \\
\text { indirectes } \\
\text { - politesse } \\
\text { - niveaux de } \\
\text { langue } \\
\text { - émoticônes } \\
\text { - etc. }\end{array}$ & \begin{tabular}{|l|} 
- lexique de la \\
correspondance \\
formelle $v s$ \\
informelle \\
- interrogatives \\
directes et \\
indirectes \\
- etc.
\end{tabular} & $\begin{array}{l}\text { - l'im/politesse } \\
\text { - les relations } \\
\text { amicales } \\
\text { - la } \\
\text { correspondan- } \\
\text { ce électronique } \\
\text { - les modes de } \\
\text { communica- } \\
\text { tion à distance } \\
\text { - etc. }\end{array}$ & $\begin{array}{l}\text { - organiser un } \\
\text { événement } \\
\text { festif }\end{array}$ \\
\hline
\end{tabular}

Tableau 1

Une telle connaissance des caractéristiques d'un genre peut donner lieu à la prise en considération d'une entrée ou d'une autre. Tout en s'appuyant sur le genre, le point de départ du formateur peut être linguistique, pragmatique, culturel, etc. en fonction des objectifs fixés eu égard aux besoins des apprenants, de l'intérêt thématique du document, du niveau du public, de sa progression, de la compétence à travailler (oral vs écrit ; compréhension vs expression) ou encore, de la tâche envisagée.

\section{Pour conclure}

En FLE, la délimitation des contenus à enseigner en objets d'enseignement croise trois spécificités, à l'écart, pour les deux premières du moins, de ce qu'on l'on observe en FL1 : l'absence de programme prédéfini, l'appui sur l'analyse des besoins des apprenants et la didactisation de documents authentiques.

Il se dégage de ces orientations la nécessaire caractérisation de l'apprenant. Aussi les préoccupations des didacticiens ont-elles été d'accorder les contenus de leur formation au plus près des besoins de ce dernier. Dans le prolongement de cette démarche, prendre appui sur des entrées énonciatives et/ou pragmatiques pour circonscrire des genres est un moyen d'identifier des pratiques scripturales intrinsèquement liées à des secteurs d'activités déterminés (comme la santé, l'éducation ou l'action sociale par exemple) et ce faisant, à des comportements socialement marqués. Une étape indispensable à mettre en œuvre dans le cadre de l'enseignement/apprentissage d'une langue étrangère pour cerner le fonctionnement des discours et choisir de façon informée des documents adaptés aux besoins et au profil de publics d'apprenants très différents. En ce sens, la démarche apporte une réponse à une demande sociale attachée à des préoccupations didactiques.

\section{Références bibliographiques}

Adam, J.-M. (1990). Eléments de linguistique textuelle. Liège : Mardaga.

Adam, J.-M. (1992a). Les textes : type et prototypes. Paris : Nathan. 
Adam, J.-M. (1992b). Linguistique textuelle, Des genres de discours aux textes. Paris : Nathan Université.

Adam, J.-M. (2002). Texte. In Charaudeau P. et Maingueneau D. (éds). Dictionnaire d'analyse du discours. Paris : Seuil, 570-572.

Austin, J.-L. (1970). Quand dire, c'est faire. Paris : Seuil, Points.

Bakhtine, M. (1977). Le marxisme et la philosophie du langage. Paris : Minuit.

Bakhtine, M. (1984). Esthétique de la création verbale. Paris : Gallimard, NRF.

Beacco, J.-C. (1980). Compétence de communication: des objectifs d'enseignement aux pratiques de classe. Le Français dans le Monde, $\mathrm{n}^{\circ} 153,35-40$.

Beacco, J.-C. (1989). Un rendez-vous manqué ? Théories du discours et grammaire en didactique du français langue étrangère. Le français dans le monde, recherches et applications, numéro spécial février-mars, 138-146.

Beacco, J.-C. (2004). Trois perspectives linguistiques sur la notion de genre discursif. Langages, ${ }^{\circ} 153$, Vol. 38, 109-119.

Beacco, J.-C. (2013). L'approche par genres discursifs dans l'enseignement du français langue étrangère et langue de scolarisation. Pratiques, n $157-158,189-200$.

Beacco, J.-C., Darot, M. (1984). Analyses de discours, Lecture et expression. Paris : Hachette/Larousse.

Béal, C. (2010). Les interactions quotidiennes en français et en anglais, De l'approche comparative à l'analyse des situations interculturelles. Bern : Peter Lang.

Benveniste, E. (1966). Problèmes de linguistique générale. Tome 1. Paris : Gallimard.

Benveniste, E. (1974). Problèmes de linguistique générale. Tome 2. Paris : Gallimard.

Besse, H. (1980a). La question fonctionnelle. in Besse, H. et Galisson, R. (ed.), Polémique en didactique, Du nouveau en question, Paris, Cle International, 30-136.

Besse, H. (1980b). De la pratique des textes non littéraires au niveau II. Le français dans le monde, n 150, 50-57.

Bronckart, J.-P. (1996). Activité langagière, textes et discours. Lausanne-Paris : Delachaux et Niestlé.

Bronckart, J.-P. (2004). Les genres de textes et leur contribution au développement psychologique. Langages, $\mathrm{n}^{\circ} 153$, 98-108.

Bronckart, J.-P., Bain, D., Schneuwly, B., Davaud, C., Pasquier, A. (1985). Le fonctionnement des discours. Neuchâtel-Paris : Delachaux et Niestlé.

Claudel, Ch. (2004). La notion de figure : propositions méthodologiques pour une approche comparée du genre interview de presse en français et en japonais. Tranel, $n^{\circ} 40,27-45$.

Claudel Ch. (2009). L'interview de presse. Les Langues Modernes, 2009-2, Disponible sur Internet, accès réservé aux abonnés : www.APLV-LanguesModernes.org

Claudel Ch. (2010a). Les pratiques journalistiques en France et au Japon : points de rencontre et divergences. Communication \& Langages, $\mathrm{n}^{\circ} 164,13-31$.

Claudel Ch. (2010b). La formule d'appel dans les courriels à caractère personnel français et japonais. Journal of French Language Studies, Vol. 20, n ${ }^{\circ} 1,31-46$.

Claudel, Ch. (2014). L'analyse de discours et la notion de genre dans la formation à la didactique des langues. In Causa, M., Galligani, S., Vlad, M. (dir.). Formation et pratiques enseignantes en contextes pluriels. Paris : Éditions Riveneuves, 91-114.

Claudel, Ch. (2015). Apologies and thanks in french and japanese personal emails: a comparison of politeness pratices. Russia Journal of Linguistics, Special Issue: Intercultural Communication: Theory and Practice, vol. 23, $\mathrm{n}^{\circ} 4,127-145$.

Claudel, Ch., Lemeunier, V. (2012). Créer une banque de données de documents authentiques : quels critères retenir ? Intercompreensão. Revista de Didáctica das Linguas, 139-160.

Conseil de l'Europe. (1976). Un Niveau-Seuil. Paris : Didier/Hatier. 
Coste, D. (1980a). Communicatif, fonctionnel, notionnel et quelques autres. Le Français dans le Monde, $\mathrm{n}^{\circ} 153,25$ 34.

Coste, D. (1980b). Analyse de discours et pragmatique de la parole dans quelques usages d'une didactique des langues. Applied Linguistics, $\mathrm{n}^{\circ} 1-3,244-252$.

Courtilon, J. (2003). Élaborer un cours de FLE. Paris : Hachette.

De Pietro, J.-F., Erard, S., Kaneman-Pougatch, M. (1996/97). Un modèle didactique du 'débat' : de l'objet social à la pratique scolaire, Enjeux, n³9/40, 100-129.

De Pietro, J.-F., Dolz, J. (1997). L'oral comme texte : comment construire un objet enseignable ? Education et recherches, $\mathrm{n}^{\circ} 19,335-359$.

De Pietro, J.-F., Schneuwly, B. (2003). Le modèle didactique du genre : un concept de l'ingénierie didactique, Les cahiers Théodile, $\mathrm{n}^{\circ} 3,27-52$.

De Pietro, J.-F., Pfeiffer Ryter, V., Wirthner, M., Beguin, M., Broi, A.-M., Clément S., Matei, A., Roos, E. (2009). Evaluation de la collection du moyen d'enseignement. S'exprimer en français - Rapport final. Neuchâtel : IRDP.

Dolz, J., Schneuwly, B. (1998). Pour un enseignement de l'oral, initiation aux genres formels à l'école. Paris : ESF éditeur.

Dolz-Mestre, J., Noverraz, M., Schneuwly, B. (éd.) (2001). S'exprimer en français : Séquences didactiques pour l'oral et pour l'écrit. Bruxelles : De Boeck.

Dolz, J., Surian M., Abouzaïd M. (2015) : Des actes de parole aux genres textuels : évolution de l'enseignement du français dans les classes d'accueil. In Defays, J.-M. et al., 20 ans de FLES - Faits et gestes de la didactique du FLE/S de 1995 à 2015. Fernelmont : Proximités EME, 123-142.

Germain, C. (1993). Évolution de l'enseignement des langues : 5000 ans d'histoire. Paris : Cle International.

Goffman, E. (1974). Les rites d'interaction. Paris : Minuit.

Grize, J.-B. (1990). Logique et langage. Paris : Orphys.

Hymes, D.-H. (1972). On Communicative Competence. In Pride J.-B. \& Holmes J.-H. (ed.). Sociolinguistics. Harmondsworth : Penguin, 269-293.

Hymes, D.-H. (1984). Vers la compétence de communication. Paris : Hatier-Crédif.

Kerbrat-Orecchioni, C. (1994). Les interactions verbales. Tome III. Paris : A. Colin.

Kerbrat-Orecchioni, C., Traverso, V. (2008). Les interactions en site commercial, Invariants et variations. Lyon : ENS Editions.

Labov, W. (1966). The social stratification of English in New York City. Washington DC: Centre for applied linguistics.

Laurens, V. (2012). Modéliser des séquences en FLE et en FL1 : analyse comparée de l'unité didactique et de la séquence didactique. Le français aujourd' $h u i, \mathrm{n}^{\circ} 176,59-75$.

Laurens, V. (2013). Formation à la méthodologie de l'enseignement du français langue étrangère et développement de l'agir enseignant. Thèse en Didactique des langues et des cultures : Université Sorbonne nouvelle-Paris 3.

Lebre-Peytard, M. (1990). Situations d'oral. Paris : Clé international.

Maingueneau, D. (2002). Analyser des textes de communication. Paris : Nathan Université.

Moirand, S. (1979). Situations d'écrit. Paris : CLE International.

Moirand, S. (1990a). Une grammaire des textes et des dialogues. Paris : Hachette.

Moirand, S. (1990b). Enseigner à communiquer en langue étrangère. Paris : Hachette.

Mourlhon-Dallies, F. (1995). Une méthodologie pour l'analyse linguistique de genres discursifs produits en situation professionnelle: étude d'écrits touristiques sur Venise en quatre langues, Thèse de doctorat de Sciences du Langage, Université Sorbonne nouvelle-Paris 3.

Mourlhon-Dallies, F. (2008). Enseigner une langue à des fins professionnelles. Paris, Didier. 
Peytard, J., Moirand, S. (1992). Discours et enseignement du français, Les lieux d'une rencontre. Paris : Hachette.

Rastier, F. (2001). L'action et le sens, Pour une sémiotique des cultures. Journal des anthropologues, ${ }^{\circ}$ 85-86, 183219.

Richterich, R. (1985). Besoins langagiers et objectifs d'apprentissage. Paris : Hachette, coll. «F. ».

Roulet, E. (1980). Langue maternelle et langues secondes, Vers une pédagogie intégrée. Paris : Crédif-Hatier.

Searle, J.-R. (1972). Les actes de langage. Paris : Hermann, Collection "Savoir".

Schneuwly, B., Dolz, J. (1997). Les genres scolaires. Des pratiques scolaires aux objets d'enseignement. Repères, $\mathrm{n}^{\circ} 15,27-40$.

Traverso, V. (2001). Interactions ordinaires dans les petits commerces : éléments pour une comparaison interculturelle. Langage \& société, $\mathrm{n}^{\circ}$ 95, 5-31.

Truong, H. L. (2010). Des perspectives didactiques de la notion de genre de discours à une approche didactique de la lecture- compréhension en FLE. In Neveu F., Muni Toke V., Durand J., Klingler T., Mondada L., Prévost S. (éds.), Congrès Mondial de Linguistique Française - CMLF 2010, 623-643. Article disponible sur le site http://www.linguistiquefrancaise.org ou http://dx.doi.org/10.1051/cmlf/2010028

Verdelhan, M. (1982). Le renouvellement des concepts en didactique et la formation des enseignants de français langue étrangère. Langue française, Vol. 55, $\mathrm{n}^{\circ} 1,105-117$.

von Münchow, P. (2004). Le journal télévisé en France et en Allemagne. Plaisir de voir ou devoir de s'informer. Paris : Presses Sorbonne Nouvelle.

von Münchow, P. (2011). Lorsque l'enfant paraît... Le discours des guides parentaux en France et en Allemagne. Toulouse : Presses Universitaires du Mirail.

\section{Manuels de FLE}

Denyer, M., Garmendia, A., Lions-Olivieiri, M.-L. (2009).Version Originale 1. Paris : Maison des langues.

Flumian, C., Labascoule, J., Liria, P., Rodriguez, M.-R., Royer, C. (2011). Nouveau Rond-Point 1. Paris : Maison des langues.

Beacco di Giura, M., Jennepin, D., Kaneman-Pougatch, M., Trevisi, S. (1997). Café Crème. Paris : Hachette.

Loiseau, Y., Mérieux, R. (2004). Connexions. Paris : Didier.

\footnotetext{
${ }^{1}$ L'application de la démarche est plus vraie pour des publics adultes précisément circonscrits que dans le cadre d'enseignements de langues généraux s'adressant à des débutants, les besoins des premiers étant moins prévisibles que ceux des seconds.

${ }^{2}$ Ce terme fait écho aux positions fonctionnalistes de la sociologie. Dans ce champ, « « [s]e trouvent mis en relation avec l'acte et l'acteur des termes comme fin, moyen, situation, ressources, conditions. Ailleurs c'est l'autonomie de l'acteur qui est discutée, au niveau des choix qu'il a à effectuer. Système, institution, collectivité, interaction, motivation, rôle, autant d'autres concepts qui ordonnent dans le modèle progressivement construit et révisé par Parsons. L'individu ne peut être envisagé indépendamment d'autrui, avec qui il interagit, ni indépendamment de la culture où ils s'inscrivent. » (Coste 1980a : 31-32) Ce positionnement, de nature fonctionnaliste, met en relief la place centrale accordée à l'individu.

3 Ces moyens d'enseignement ont été publiés en 4 volumes, regroupant 35 séquences didactiques, pour l'enseignement du français en Suisse romande (dans le cadre de la scolarité obligatoire), sous l'égide de la Commission romande des moyens d'enseignement (COROME). Le propos global de ces manuels était d'outiller les enseignants sur le plan des démarches et des matériaux didactiques et pédagogiques afin de promouvoir un
} 
enseignement de l'expression orale et de l'expression écrite par des genres textuels spécifiques (tels le récit de vie, l'article encyclopédique, l'exposé oral, l'interview, etc.) (De Pietro et al. 2009).

${ }^{4}$ Nous ne reviendrons pas ici sur le débat relatif au bien fondé de l'introduction de supports authentiques dans un contexte de diffusion différent de son contexte «naturel » d'émergence (cf. Besse 1980b).

${ }^{5}$ Cette étape préalable de la réflexion ne doit cependant pas occulter l'importance d'une articulation avec des extérieurs linguistiques comme le type de support (quotidien, hebdomadaire, médias grand public, spécialisé, etc.), le profil de la cible, le positionnement idéologique, etc. Autant d'aspects plus spécifiques de la culture de laquelle émanent les documents.

${ }^{6}$ Elle peut être déclinée sous de multiples formes : de rupture, d'amour, de réclamation, de déclaration de sinistre, amicale, etc.

${ }^{7}$ Cf. Alter Ego 3 B1 (Dollez et Pons 2006) ; Edito B2 (Heu et Mabilat 2006). 